Pacific Journal of Mathematics

SOME ABSTRACT GENERALIZATIONS OF THE
LJUSTERNIK-SCHNIRELMANN-BORSUK COVERING

THEOREM 


\section{SOME ABSTRACT GENERALIZATIONS OF THE LJUSTERNIK-SCHNIRELMANN-BORSUK COVERING THEOREM}

\section{H. Steinlein}

Ljusternik and Schnirelmann and independently Borsuk proved the following well known result: Let $H_{1}, \cdots, H_{k}$ be closed subsets of the sphere $S^{n}$ such that $\bigcup_{i=1}^{k} H_{i}=S^{n}$ and $H_{i} \cap\left(-H_{i}\right)=\varnothing$ for $i=1, \cdots, k$, then $k \geqq n+2$.

In this paper, this result is considered from an abstract topological viewpoint: We develope methods for the proof of generalizations of this result in the context of the genus in the sense of A. S. Šarc.

1. Introduction The main concept, which is used in this paper, is the "genus" in the sense of A. S. Švarc (cf. [6, 7]).

DEFINITION 1. (cf. $[6,7,8]$; for another way to introduce this notion cf. [6, 7].) Let $M$ be a topological Hausdorff space, $p$ a prime number and $f: M \rightarrow M$ a free $\boldsymbol{Z}_{p}$-action (i.e., $f$ is continuous, $f^{p}=i d$ and $f(x) \neq x$ for all $x \in M)$. Then

$\mathscr{C}(M, f):=\left\{G \subset M \mid\right.$ There exist disjoint closed sets $G_{0}, \cdots, G_{p-1} \subset M$ with $\bigcup_{i=0}^{p-1} G_{i}=G$ and $f^{i}\left(G_{0}\right)=G_{i}$ for $\left.i=1, \cdots, p-1\right\}$,

and the genus $g(M, f)$ is defined by

$$
g(M, f):=\min \{\operatorname{card} \mathscr{G} \mid \mathscr{G} \subset \mathscr{C}(M, f), \quad \cup \mathscr{G}=M\} .
$$

The genus has several very nice properties (cf. $[6,7,8]$ ). It is closely related to the earlier notions of the Ljusternik-Schnirelmann category [5] and the Yang index [9]. In general, it is difficult to compute the genus, but there are various estimates in terms of the dimension, connectivity, or (co-)homology of the space.

As for the Ljusternik-Schnirelmann-Borsuk result, it is interesting that, independently of the prime number $p$ and the action $f$, we always have $g\left(S^{n}, f\right)=n+1$ (this result is mainly due to Krasnosel'skii [4]). Thus, in the Ljusternik-Schnirelmann-Borsuk theorem, we could replace the estimate $k \geqq n+2$ by $k \geqq$ $g\left(S^{n},-i d\right)+1$, and with this estimate, the result holds in a trivial way in a much more general setting.

THEOREM. (cf. $[9,8]$ ) Let $M$ be a Hausdorff space, $f: M \rightarrow M a$ free $\boldsymbol{Z}_{2}$-action (i.e., a fixed-point-free involution) and let $M_{1}, \cdots, M_{k} \subset M$ 
be closed sets such that $\bigcup_{i=1}^{k} M_{i}=M$ and $M_{i} \cap f\left(M_{i}\right)=\varnothing$ for $i=$ $1, \cdots, k$. Then $k \geqq g(M, f)+1$.

On the other hand, the analogous question for $\boldsymbol{Z}_{p}$-actions with $p \geqq 3$ seems to be much more complicated. I formulate it only for normal spaces, since I have no idea how one could treat the general case of Hausdorff spaces.

Problem 1. Let $M$ be a normal topological space, $p \geqq 3$ a prime number, $f: M \rightarrow M$ a free $Z_{p}$-action and $M_{1}, \cdots, M_{k} \subset M$ closed sets such that $\mathrm{U}_{i=1}^{k} M_{i}=M$ and $M_{i} \cap f\left(M_{i}\right)=\varnothing$ for $i=1, \cdots, k$. What is the best estimate of $g(M, f)$ in terms of $k$ and $p$ ?

There is some motivation for this problem. If one could prove an estimate $g(M, f) \leqq r(k, p)$ with $r(k, p)=o(p)$ for every fixed $k$, this would imply that the following long standing conjecture in asymptotic fixed point theory is true (cf. [8]).

Conjecture. Let $E$ be a normed space, $H \subset E$ a nonempty closed convex set and $f: H \rightarrow H$ a continuous map such that $f^{m_{0}}(H)$ is relatively compact for some $m_{0} \in N$. Then $f$ has a fixed point (?).

At present, instead of the needed $o(p)$-estimate, only a $O(p)$ estimate is known: In [8], $g(M, f) \leqq(p-1) / 2(k-2)$ was proved for compact spaces $M$, a result which will be slightly improved in this paper.

The main result of this paper (Theorem 2) is a reduction of Problem 1 to the equivalent problem of computing the genus of nice space $L_{k, p}$ with nice actions $\varphi_{k, p}$ on it. It will be shown that $g(M, f) \leqq g\left(L_{k, p}, \varphi_{k, p}\right)$, where $\left(L_{k, p}, \varphi_{k, p}\right)$ is a prototype for $(M, f)$ in Problem 1.

To date, only for $p=2$ or for $k=3$ have the values of $g\left(L_{k, p}, \varphi_{k, p}\right)$ been computed and only rough estimates are available for the general case. But the spaces $L_{k, p}$ and the actions $\varphi_{k, p}$ seem to be nice enough to allow numerical computations of $g\left(L_{k, p}, \varphi_{k, p}\right)$ for small numbers $k$ and $p$ (e.g., $k, p \leqq 7$ ), which might suggest the general result one should expect. My own (a little vage) conjecture is $g\left(L_{k, p}, \varphi_{k, p}\right)=k-s(k, p)$ with $s(k, p) \in\{1,2,3\}$.

2. The reduction of Problem 1. Let $N:=\{1,2,3, \cdots\}$ and $\boldsymbol{R}^{\infty}:=\{x: \boldsymbol{N} \rightarrow \boldsymbol{R} \mid x(n)=0$ for almost every $n \in N\}$, equipped with the usual Euclidean topology. Let $E_{i} \in \boldsymbol{R}^{\infty}, E_{i}(n):=\delta_{i n}$ for all $n \in N$, 
and for $q \in N, I \subset\{1, \cdots, q\}$ and $i \in\{1, \cdots, q\}$ let

$$
\begin{aligned}
& \Delta_{q-1}:=\operatorname{co}\left\{E_{1}, \cdots, E_{q}\right\}, \\
& \Delta_{q-1}^{I}:=\operatorname{co}\left\{E_{j} \mid j \in I\right\}, \\
& \Delta_{q-1 ; i}:=\Delta_{q-1}^{\{1, \cdots, q \backslash \backslash i\}}=\operatorname{co}\left\{E_{j} \mid j \in\{1, \cdots, q\} \backslash\{i\}\right\}, \\
& \partial \Delta_{q-1}:=\bigcup_{i=1}^{q} \Delta_{q-1 ; i} .
\end{aligned}
$$

Thus $\Delta_{q-1}$ is the closed $(q-1)$-dimensional simplex spanned by $E_{1}, \cdots, E_{q}$ and $\Delta_{q-1}^{I}$ and $\Delta_{q-1 ; i}$ are (closed) faces of $\Delta_{q-1}$. We denote by $[\sigma]$ the barycenter of a simplex $\sigma$.

Now we are able to state our first theorem:

THeOREM 1. Let $M$ be a normal space, $k \in N, p$ a prime number, $f: M \rightarrow M$ a free $Z_{p}$-action, and $M_{1}, \cdots, M_{k} \subset M$ closed sets such that $\bigcup_{i=1}^{k} M_{i}=M$ and $M_{i} \cap f\left(M_{i}\right)=\varnothing$ for $i=1, \cdots, k$. Then there exists a continuous map $h: M \rightarrow \partial \Delta_{k-1}$ such that $h\left(M_{i}\right) \subset \Delta_{k-1 ; i}$ and

$$
h\left(f\left(h^{-1}\left(\Delta_{k-1 ; i}\right)\right)\right) \subset \bigcup_{\substack{j=1 \\ j \neq i}}^{k} \operatorname{co}\left\{\left[\Delta_{k-1}^{K}\right] \mid\{i\} \subset K \subset\{1, \cdots, k\} \backslash\{j\}\right\},
$$

in particular $h\left(f\left(h^{-1}\left(\Delta_{k-1 ; i}\right)\right)\right) \cap \Delta_{k-1 ; i}=\varnothing$ for $i=1, \cdots, k$.

Proof. Because of $M_{i} \cap f\left(M_{i}\right)=\varnothing$ and the normality of the space $M$, there exist open $N_{i} \subset M$ with $M_{i} \subset N_{i}$ and $N_{i} \cap f\left(N_{i}\right)=\varnothing$ $(i=1, \cdots, k)$. For $I, J \subset\{1, \cdots, k\}$, let $W_{I, J}:=\bigcap_{i \in\{1, \cdots, k\} \backslash I} M_{i} \backslash \bigcup_{j \in J} N_{j}$.

We want to define $h: M \rightarrow \partial \Delta_{k-1}$ such that for $\varnothing \neq J \subset I \subset$ $\{1, \cdots, k\}$ we have

$$
h\left(W_{I, J}\right) \subset \operatorname{co}\left\{\left[\Delta_{k-1}^{K}\right] \mid J \subset K \subset I\right\}
$$

(i.e., roughly speaking, $h$ maps $W_{I, J}$ into the traverse $\operatorname{Tr}\left(\Delta_{k-1}^{J}\right)$ in the complex $\Delta_{k-1}^{I}$; cf. [2]). The existence of such a map $h$ can be proved as follows:

We proceed by induction on card $I$, starting with the trivial case card $I=0$, i.e., $I=\varnothing$. In this case we have $J=\varnothing$ and hence

$$
W_{I, J}=\bigcap_{i \in\{1, \ldots, k\}} M_{i}=\varnothing
$$

(observe that $f\left(\bigcap_{i \in\{1, \ldots, k\}} M_{i}\right) \cap M_{j} \subset f\left(M_{j}\right) \cap M_{j}=\varnothing$ for every $j \in$ $\{1, \cdots, k\}$ and hence $\left.\bigcap_{i \in\{1, \cdots, k\}} M_{i}=\varnothing\right)$.

Let $n \in\{0, \cdots, k-2\}$ and assume that we could define $h$ on

$$
M^{(n)}:=\bigcup_{\substack{I \subset\{1, \ldots, k\} \\ \text { card } I \leqq n}} \bigcap_{i \in\{1, \ldots, k\} \backslash I} M_{i}
$$


such that (1) holds for $\varnothing \neq J \subset I \subset\{1, \cdots, k\}$ with card $I \leqq n$ and such that $h$ is continuous on $M^{(n)}$.

Since for $I_{1}, I_{2} \subset\{1, \cdots, k\}$ with $I_{1} \neq I_{2}$ and $\operatorname{card} I_{1}=\operatorname{card} I_{2}=$ $n+1$, we have

$$
\bigcap_{i \in\left\{1, \ldots, k \backslash I_{1}\right.} M_{\imath} \cap \bigcap_{i \in\{1, \ldots, k\} \backslash I_{2}} M_{i}=\bigcap_{i \in\{1, \ldots, k\} \backslash\left(I_{1} \cap I_{2}\right)} M_{i} \subset M^{(x)},
$$

it suffices to extend $h$ independently to all the sets $M^{(n)} U$ $\bigcap_{i \in\{1, \ldots, k\} \backslash I} M_{i}$ with card $I=n+1$ according to our conditions. The union of all these extensions will be an extension of $h$ to $M^{(n+1)}$ with all the desired properties.

Thus we choose a fixed $I_{0} \subset\{1, \cdots, k\}$ with card $I_{0}=n+1$. We define the extension of $h$ to $M^{(n)} \cup \bigcap_{i \in\{1, \cdots, k\} \backslash I_{0}} M_{i}$ by induction on card $J$, where $J \subset I_{0}$ : We start with card $J=n+1$, i.e., $J=I_{0}$, and define

$$
h(x):=\left[\Delta_{k-1}^{I_{0}}\right] \text { for all } x \in W_{I_{0}, I_{0}} .
$$

Since $M^{(n)} \cap W_{I_{0}, I_{0}}=\varnothing$, this extension is justified and of course continuous.

Let $m \in\{2, \cdots, n+1\}$ and assume that we have defined $h$ on

$$
M_{I_{0}}^{(m)}:=M^{(n)} \cup \underset{\substack{J \subset I_{0} \\ \text { card } J \geqq m}}{\bigcup} W_{I_{0}, J}
$$

such that (1) holds for all $\varnothing \neq J \subset I \subset\{1, \cdots, k\}$ with card $I \leqq n$ or card $J \geqq m$ and $I=I_{0}$ and such that $h$ is continuous on $M_{I_{0}}^{(m)}$.

Since for $J_{1}, J_{2} \subset I_{0}$ with $J_{1} \neq J_{2}$ and $\operatorname{card} J_{1}=\operatorname{card} J_{2}=m-1$ we have

$$
W_{I_{0}, J_{1}} \cap W_{I_{0}, J_{2}}=W_{I_{0}, J_{1} \cup J_{2}} \subset M_{I_{0}}^{(m)},
$$

it suffices to extend $h$ independently to all the sets $M_{I_{0}}^{(m)} \cup W_{I_{0}, J}$ with card $J=m-1$ according to our conditions. The union of all these extensions will be an extension of $h$ to $M_{I_{0}}^{(m-1)}$ with all the desired properties.

Accordingly, let $J_{0} \subset I_{0}$ with $\operatorname{card} J_{0}=m-1$. Then we have

$$
\begin{aligned}
& W_{I_{0}, J_{0}} \cap M_{I_{0}}^{(m)} \\
& =W_{I_{0}, J_{0}} \cap\left(M^{(n)} \cup \underset{\substack{J \subset I_{0} \\
\operatorname{card} J \geqq m}}{\bigcup} W_{I_{0}, J}\right) \\
& =\left(W_{I_{0}, J_{0}} \cap M^{(n)}\right) \cup \underset{\substack{J \subset I_{0} \\
\text { card } J \geqq m}}{\bigcup}\left(W_{I_{0}, J_{0}} \cap W_{I_{0}, J}\right) \\
& =\bigcup_{\substack{I \subset\{1, \ldots \ldots, k\} \\
\operatorname{card} I \leqq n}}^{\bigcup}\left(W_{I_{0}, J_{0}} \cap \bigcap_{i \in\{1, \ldots, k\} \backslash} M_{i}\right) \cup \underset{\substack{J \subset I_{0} \\
\operatorname{card} J \geqq m}}{\bigcup} W_{I_{0}, J \cup J_{0}}
\end{aligned}
$$




$$
\begin{aligned}
& =\bigcup_{\substack{I \subset\{1, \ldots, k\} \\
\text { card } I \leqq n}} W_{I \cap I_{0}, J_{0}} \cup \underset{\substack{J \subset I_{0} \\
\text { card } J \geqq m}}{\bigcup} W_{I_{0}, J \cup J_{0}}
\end{aligned}
$$

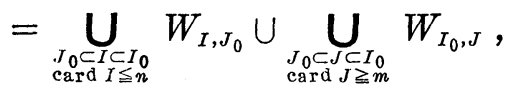

and hence

$$
\begin{aligned}
& h\left(W_{I_{0}, J_{0}} \cap M_{I_{0}}^{(m)}\right)=\bigcup_{\substack{J 0 I \subset I_{0} \\
\text { card } I \leqq n}} h\left(W_{I, J_{0}}\right) \cup \underset{\substack{J_{0} \subset J \subset I I_{0} \\
\text { card } J \geqq m}}{\bigcup} h\left(W_{I_{0}, J}\right) \\
& \subset \underset{\substack{J 0 \\
\text { card } I \leqq n}}{\bigcup} \operatorname{co}\left\{\left[\Delta_{k-1}^{K}\right] \mid J_{0} \subset K \subset I\right\} \cup \underset{\substack{J 0 \subset J \subset I_{0} \\
\operatorname{card} J \geqq m}}{\bigcup} \operatorname{co}\left\{\left[\Delta_{k-1}^{K}\right] \mid J \subset K \subset I_{0}\right\} \\
& \subset \operatorname{co}\left\{\left[\Delta_{k-1}^{K}\right] \mid J_{0} \subset K \subset I_{0}\right\} \text {. }
\end{aligned}
$$

Since every closed convex subset of a finite dimensional normed space is an $\mathrm{AR}$ (normal), we can extend $\left.h\right|_{W_{I_{0}, J_{0} \cap M} \cap M_{0}} ^{(m)}$ continuously to $W_{I_{0}, J_{0}}$ such that

$$
h\left(W_{I_{0}, J_{0}}\right) \subset \operatorname{co}\left\{\left[\Delta_{k-1}^{K}\right] \mid J_{0} \subset K \subset I_{0}\right\} .
$$

By this iterative construction, we finally obtain an extension of $h$ to the set $M_{I_{0}}^{(1)}$, which is equal to $M^{(n)} \cup \bigcap_{i \in\{1, \cdots, k\} \backslash I_{0}} M_{i}$, since for every $x \in M$ there is a $j \in\{1, \cdots, k\}$ with $x \notin N_{j}$.

This shows that we can extend $h$ continuously to $M^{(n+1)}$ such that (1) holds for $\varnothing \neq J \subset I \subset\{1, \cdots, k\}$ with card $I \leqq n+1$ and such that

$$
\begin{aligned}
& h\left(M^{(n+1)}\right) \subset \bigcup_{\substack{I \subset\{1, \ldots \ldots, k\} \\
\operatorname{card} I \leqq n+1}} \bigcup_{\varnothing \neq J \subset I} \operatorname{co}\left\{\left[\Delta_{k-1}^{K}\right] \mid J \subset K \subset I\right\}
\end{aligned}
$$

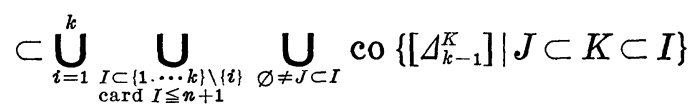

$$
\begin{aligned}
& \subset \bigcup_{i=1}^{k} \Delta_{k-1: i}=\partial \Delta_{k-1} \text {. }
\end{aligned}
$$

Thus we have proved the existence of a continuous map $h: M \rightarrow \partial \Delta_{k-1}$, which fulfills (1) for all $\varnothing \neq J \subset I \subset\{1, \cdots, k\}$. We have to prove that (1) implies $h\left(M_{i}\right) \subset \Delta_{k-1: i}$ and

$$
h\left(f\left(h^{-1}\left(\Delta_{k-1: i}\right)\right)\right) \subset \bigcup_{\substack{j=1 \\ j \neq i}}^{k} \operatorname{co}\left\{\left[\Delta_{k-1}^{K}\right] \mid\{i\} \subset K \subset\{1, \cdots, k\} \backslash\{j\}\right\}
$$

for $i=1, \cdots, k$.

Let $I_{i}:=\{1, \cdots, k\} \backslash\{i\}$. Then we have

$$
\begin{aligned}
h\left(M_{i}\right) & \subset \bigcup_{\varnothing \neq J \subset I_{i}} h\left(W_{I_{i}, J}\right) \subset \bigcup_{\varnothing \neq J \subset I_{i}} \operatorname{co}\left\{\left[\Delta_{k-1}^{K}\right] \mid J \subset K \subset I_{i}\right\} \\
& \subset \Delta_{k-1}^{T_{i}}=\Delta_{k-1 ; i} .
\end{aligned}
$$

In addition, 


$$
\begin{aligned}
h\left(M \backslash N_{i}\right) & =\bigcup_{\substack{j=1 \\
j \neq i}}^{k} h\left(M_{j} \backslash N_{i}\right) \\
& \subset \bigcup_{\substack{j=1 \\
j \neq i}}^{k} \operatorname{co}\left\{\left[\Delta_{k-1}^{K}\right] \mid\{i\} \subset K \subset\{1, \cdots, k\} \backslash\{j\}\right\} \subset \partial \Delta_{k-1} \backslash \Delta_{k-1 ; i}
\end{aligned}
$$

and hence

$$
\begin{aligned}
& h\left(f\left(h^{-1}\left(\Delta_{k-1 ; i}\right)\right)\right) \subset h\left(f\left(N_{i}\right)\right) \subset h\left(M \backslash N_{i}\right) \\
& \quad \subset \bigcup_{\substack{j=1 \\
j \neq i}}^{k} \cos \left\{\left[\Delta_{k-1}^{K}\right] \mid\{i\} \subset K \subset\{1, \cdots, k\} \backslash\{j\}\right\} .
\end{aligned}
$$

For every $k \in N$ and every prime number $p$ we define

$$
\begin{aligned}
L_{k, p}:= & \left\{\left(x_{1}, \cdots, x_{p}\right) \in\left(\partial \Delta_{k-1}\right)^{p} \mid \text { If } m, n \in\{1, \cdots, p\}, n \equiv m+1(\bmod p)\right. \\
& \text { and } \left.x_{m} \in \Delta_{k-1 ; i}, \text { then } x_{n} \notin \Delta_{k-1: i}\right\}
\end{aligned}
$$

and

$$
\begin{aligned}
\widetilde{L}_{k, p}:= & \left\{\left(x_{1}, \cdots, x_{p}\right) \in\left(\partial \Delta_{k-1}\right)^{p} \mid \text { If } m, n \in\{1, \cdots, p\}, n \equiv m+1(\bmod p)\right. \\
& \text { and } x_{m} \in \Delta_{k-1 ; i}, \text { then } \\
& \left.x_{n} \in \bigcup_{\substack{j=1 \\
j \neq i}}^{k} \operatorname{co}\left\{\left[\Delta_{k-1}^{K}\right] \mid\{i\} \subset K \subset\{1, \cdots, k\} \backslash\{j\}\right\}\right\} .
\end{aligned}
$$

Obviously, $\tilde{L}_{k, p} \subset L_{k, p}$, and the $\operatorname{map} \varphi_{k, p}: L_{k, p} \rightarrow L_{k, p}, \varphi_{k, p}\left(x_{1}, \cdots, x_{p}\right):=$ $\left(x_{2}, \cdots, x_{p}, x_{1}\right)$ is a free $\boldsymbol{Z}_{p}$-action on $L_{k, p}$ and on $\widetilde{L}_{k, p}$. Now we can prove

THEOREM 2. Let $M$ be a normal space, $k \in N, p$ a prime number and $f: M \rightarrow M$ a free $Z_{p}$-action. Let $M_{1}, \cdots, M_{k} \subset M$ be closed sets such that $\mathrm{U}_{i=1}^{k} M_{i}=M$ and $M_{i} \cap f\left(M_{i}\right)=\varnothing$ for $i=1, \cdots, k$. Then we have $g(M, f) \leqq g\left(\widetilde{L}_{k, p}, \varphi_{k, p}\right)=g\left(L_{k, p}, \varphi_{k, p}\right)$.

Proof. By Theorem 1, there exists a continuous map $h: M \rightarrow \partial \Delta_{k-1}$ such that $h\left(M_{i}\right) \subset \Delta_{k-1 ; i}$ and such that

$$
\begin{aligned}
h\left(f\left(h^{-1}\left(\Delta_{k-1: i}\right)\right)\right) & \subset \bigcup_{\substack{j=1 \\
j \neq i}}^{k} \operatorname{co}\left\{\left[\Delta_{k-1}^{K}\right] \mid\{i\} \subset K \subset\{1, \cdots, k\} \backslash\{j\}\right\} \\
& \subset \partial \Delta_{k-1} \backslash \Delta_{k-1 ; i} .
\end{aligned}
$$

Let $P: M \rightarrow \widetilde{L}_{k, p}, \quad P(x):=\left(h(x), h(f(x)), \cdots, h\left(f^{p-1}(x)\right)\right)$. Obviously, $P$ is an equivariant map (i.e., $P \circ f=\varphi_{k, p} \circ P$ ) and hence $g(M, f) \leqq$ $g\left(\widetilde{L}_{k, p}, \varphi_{k, p}\right) \leqq g\left(L_{k, p}, \varphi_{k, p}\right)$ (cf. $\left.[7,8]\right)$.

Conversely, $g\left(L_{k, p}, \varphi_{k, p}\right) \leqq g\left(\widetilde{L}_{k, p}, \varphi_{k, p}\right)$ follows from the fact that $L_{k, p}$ can be covered by the closed subsets $\hat{M}_{i}:=\left\{\left(x_{1}, \cdots, x_{p}\right) \in\right.$ $\left.L_{k, p} \mid x_{1} \in \Delta_{k-1 ; i}\right\} \quad(i=1, \cdots, k)$, which obviously have the property 
$\widehat{M}_{i} \cap \varphi_{k, p}\left(\hat{M}_{i}\right)=\varnothing$, and hence the estimate $g(M, f) \leqq g\left(\widetilde{L}_{k, p}, \varphi_{k, p}\right)$ applies to $\left(L_{k, p}, \varphi_{k, p}\right)$ instead of $(M, f)$.

REMarks. 1. Theorem 2 reduces Problem 1 to the following equivalent problem:

Problem 2. Let $k \in N$ and $p$ a prime number. What is the value of $g\left(L_{k, p}, \varphi_{k, p}\right)=g\left(\widetilde{L}_{k, p}, \varphi_{k, p}\right)$ ?

The end of the proof of Theorem 2 shows that, in fact, the value of $g\left(L_{k, p}, \varphi_{k, p}\right)$ gives the best estimate for $g(M, f)$.

2. Since the $\widetilde{L}_{k, p}$ are finite dimensional compact sets, Theorem 2 shows that for Problem 1 one cannot expect a better estimate for finite dimensional compact spaces $M$ than for the larger class of normal spaces.

3. Computing $g\left(L_{k, p}, \varphi_{k, p}\right):$ First results. I can give here the exact value of $g\left(L_{k, p}, \varphi_{k, p}\right)$ only for the special cases $p=2$ and $k=3$. For the rest, only rough estimates are available.

TheOREM 3. (cf. [9] and [8], Satz 8.) Let $k \in N$. Then $g\left(L_{k, 2}, \varphi_{k, 2}\right)=k-1$.

Proof. Let $M_{i}:=\left\{\left(x_{1}, x_{2}\right) \in L_{k, 2} \mid x_{1} \in \Delta_{k-1 ; i}\right\}(i=1, \cdots, k)$. Then we have $M_{i} \cap \varphi_{k, 2}\left(M_{i}\right)=\varnothing$ and hence $M_{k} \subset \bigcup_{i=1}^{k-1} \varphi_{k, 2}\left(M_{i}\right)$, which implies

$$
L_{k, 2}=\bigcup_{i=1}^{k} M_{i}=\bigcup_{i=1}^{k-1} M_{i} \cup M_{k}=\bigcup_{i=1}^{k-1}\left(M_{i} \cup \varphi_{k, 2}\left(M_{i}\right)\right) \text {. }
$$

Since $M_{i} \cup \varphi_{k, 2}\left(M_{i}\right) \in \mathscr{C}\left(L_{k, 2}, \varphi_{k, 2}\right)$, we have $g\left(L_{k, 2}, \varphi_{k, 2}\right) \leqq k-1$.

It is a well known fact that the sphere $S^{k-2}$ can be covered by closed sets $M_{1}, \cdots, M_{k}$ such that $M_{i} \cap\left(-M_{i}\right)=\varnothing$ for $i=1, \cdots, k$ (cf. [1]). Thus, by Theorem 2 we have $g\left(L_{k, 2}, \varphi_{k, 2}\right) \geqq g\left(S^{k-2},-i d\right)=$ $k-1$.

A less trivial result is

THEOREM 4. Let $p \geqq 3$ be a prime number. Then

$$
g\left(L_{3, p}, \varphi_{3, p}\right)=\left\{\begin{array}{lll}
1 & \text { if } & p=3 \\
2 & \text { if } & p \geqq 5
\end{array} .\right.
$$

Proof. I. Obviously, $L_{3,3} \neq \varnothing$ and hence $g\left(L_{3,3}, \varphi_{3,3}\right) \geqq 1$. On the other hand, for every $x \in L_{3,3}$, the set $M_{1}:=\left\{\left(x_{1}, x_{2}, x_{3}\right) \in L_{3,3} \mid x_{1} \in \Delta_{2: 1}\right\}$ contains exactly one of the points $x, \varphi_{3,3}(x), \varphi_{3,3}^{2}(x)$, which shows 
that $\varphi_{3,3}^{j}\left(M_{1}\right) \cap \varphi_{3,3}^{k}\left(M_{1}\right)=\varnothing$ for $j, k=0,1,2, j \neq k$ and $\bigcup_{j=0}^{2} \varphi_{3,3}^{j}\left(M_{1}\right)=$ $L_{3,3}$. Hence $g\left(L_{3,3}, \varphi_{3,3}\right) \leqq 1$.

II. Let $p \geqq 5$. To show that $g\left(L_{3, p}, \varphi_{3, p}\right) \geqq 2$, we consider the space $S^{1}(\subset C)$ with the $\boldsymbol{Z}_{p}$-action $f: S^{1} \rightarrow S^{1}, f(z):=e^{((p-1) / p) \pi i} z$. We cover $S^{1}$ by the sets $M_{j}:=\left\{e^{i \alpha} \mid 2 \pi(j-1) / 3 \leqq \alpha \leqq 2 \pi j / 3\right\}$ for $j=1,2,3$. By the definition of $f$, it follows that $M_{j} \cap f\left(M_{j}\right)=\varnothing$. Hence, by Theorem 2, we have $2=g\left(S^{1}, f\right) \leqq g\left(L_{3, p}, \varphi_{3, p}\right)$.

It remains to prove that $g\left(L_{3, p}, \varphi_{3, p}\right) \leqq 2$. For every $x=$ $\left(x_{1}, \cdots, x_{p}\right) \in L_{3, p}$, we define

$$
T_{x}:=\left\{\left(a_{1}, \cdots, a_{p}\right) \in\{1,2,3\}^{p} \mid x_{j} \in \Delta_{2 ; a_{j}} \text { for } j=1, \cdots, p\right\} .
$$

For $a, b \in\{1,2,3\}, a \neq b$, let

$$
r(a, b):=\left\{\begin{array}{lll}
1 & \text { if } & b \equiv a+1(\bmod 3) \\
2 & \text { if } & b \equiv a+2(\bmod 3)
\end{array}\right.
$$

and for each $j \in\{1, \cdots, p\}$, let

$$
j^{+}:=\left\{\begin{array}{ll}
j+1 & \text { if } j \leqq p-1 \\
1 & \text { if } j=p
\end{array} \text { and } j^{-}:= \begin{cases}j-1 & \text { if } j \geqq 2 \\
p & \text { if } j=1\end{cases}\right.
$$

Then, for $x \in L_{3, p}$, we define

$$
v(x):=\frac{1}{3} \sum_{i=1}^{p} r\left(a_{j}, a_{j^{+}}\right),
$$

where $\left(a_{1}, \cdots, a_{p}\right)$ is an arbitrary element of $T_{x}$. We have to show that this definition does not depend on the special choice of $\left(a_{1}, \cdots, a_{p}\right) \in T_{x}$. Let $\left(a_{1}, \cdots, a_{p}\right),\left(b_{1}, \cdots, b_{p}\right) \in T_{x}$ and let $j_{1}, \cdots, j_{l} \in$ $\{1, \cdots, p\}$ with $j_{1}<j_{2}<\cdots<j_{l}$ such that $a_{j_{k}} \neq b_{j_{k}}$ for $k=1, \cdots, l$, but $a_{j}=b_{j}$ for $j \in\{1, \cdots, p\} \backslash\left\{j_{1}, \cdots, j_{l}\right\}$. Then, by the definition of $L_{3, p}$, we have

$$
a_{j_{k}^{+}}=a_{j_{k}^{-}}=b_{j_{k}^{+}}=b_{j_{k}^{-}} \in\{1,2,3\} \backslash\left\{a_{j_{k}}, b_{j_{k}}\right\} \text { for } k=1, \cdots, l .
$$

Hence we have, setting $J:=\left\{j_{1}^{-}, \cdots, j_{l}^{-}, j_{1}, \cdots, j_{l}\right\}$,

$$
\begin{aligned}
\frac{1}{3} \sum_{j=1}^{p} r\left(a_{j}, a_{j^{+}}\right) & =\frac{1}{3} \sum_{j \in\{1, \ldots, p\} \backslash J} r\left(a_{j}, a_{j^{+}}\right)+l \\
& =\frac{1}{3} \sum_{j \in\{1, \ldots, p\} \backslash J} r\left(b_{j}, b_{j^{+}}\right)+l=\frac{1}{3} \sum_{j=1}^{p} r\left(b_{j}, b_{j^{+}}\right) .
\end{aligned}
$$

Obviously, $v(x) \in N, \quad p / 3 \leqq v(x) \leqq 2 p / 3$ and $v(x)=v\left(\varphi_{3, p}(x)\right)$ for all $x \in L_{3, p}$. Furthermore, all the sets $W_{n}:=v^{-1}(n)(n \in N)$ are closed. Since $L_{3, p}$ is the finite, disjoint union of the closed sets $W_{n}(n \in N$, $p / 3 \leqq n \leqq 2 p / 3)$, which are invariant under $\varphi_{3, p}$, it suffices to show that $g\left(W_{n}, \varphi_{3, p}\right) \leqq 2$ for all $n \in N, p / 3 \leqq n \leqq 2 p / 3$. 
We assume that there exists such an $n$ with $g\left(W_{n}, \varphi_{3, p}\right) \geqq 3$. Without loss of generality, we may assume that $g\left(W_{n}, \varphi_{3, p}\right)=3$, otherwise we could replace $W_{n}$ by a subset $\widetilde{W}_{n}$ with $\varphi_{3, p}\left(\widetilde{W}_{n}\right)=\widetilde{W}_{n}$ and $g\left(\widetilde{W}_{n}, \varphi_{3, p}\right)=3$.

Let $h: \partial A_{2} \rightarrow S^{1}(\subset C)$ be a homeomorphism such that

$$
h\left(\Delta_{2: j}\right)=\left\{e^{i \alpha} \mid(j-1) \frac{2 \pi}{3} \leqq \alpha \leqq j \frac{2 \pi}{3}\right\} \quad \text { for } \quad j=1,2,3 .
$$

We want to construct a map $P: W_{n} \rightarrow S^{1}$ via a homotopy argument, such that $P$ is equivariant with respect to $\varphi_{3, p}$ and $f: S^{1} \rightarrow S^{1}$,

$$
f(z):=e^{((2 \pi i) / p) n} z \text {, i.e., } P\left(\varphi_{3, p}(x)\right)=e^{(2 \pi i) / p) n} P(x)=f(P(x))
$$

for all $x \in W_{n}$. This will imply that $g\left(W_{n}, \varphi_{3, p}\right) \leqq g\left(S^{1}, f\right)=2$ in contradiction to $g\left(W_{n}, \varphi_{3, p}\right)=3$ (cf. [7] and [8], Hilfssatz 10).

Since $g\left(W_{n}, \varphi_{3, p}\right)=3$, there exist closed subsets $W_{n}^{(j, k)}, W_{n}^{(j)}$ $(j=1,2,3 ; k=0, \cdots, p-1)$ such that $W_{n}^{(j)}=\bigcup_{k=0}^{p-1} W_{n}^{(j, k)}, \bigcup_{j=1}^{3} W_{n}^{(j)}=$ $W_{n}, \quad W_{n}^{\left(j, k_{1}\right)} \cap W_{n}^{\left(j, k_{2}\right)}=\varnothing \quad$ for $\quad k_{1}, k_{2}=0, \cdots, p-1, \quad k_{1} \neq k_{2} \quad$ and $\varphi_{3, p}^{k}\left(W_{n}^{(j, 0)}\right)=W_{n}^{(j, k)}$ for $k=1, \cdots, p-1 \quad(j=1,2,3)$. We have to construct a special homotopy

$$
H:\left(W_{n}^{(1)} \cup W_{n}^{(2)} \cup W_{n}^{(3.0)}\right) \times[0,1] \longrightarrow S^{1}:
$$

(a) We define

$$
\begin{aligned}
& H(x, t):=h\left(x_{1}\right) \text { for }(x, t)=\left(\left(x_{1}, \cdots, x_{p}\right), t\right) \\
& \in\left(\left(W_{n}^{(1)} \cup W_{n}^{(2)} \cup W_{n}^{(3,0)}\right) \times\{0\}\right) \cup\left(W_{n}^{(1,0)} \times[0,1]\right),
\end{aligned}
$$

and

$$
H(x, 1):=f^{k}\left(H\left(\varphi_{3, p}^{p-k}(x), 1\right)\right)=e^{((2 \pi i) / p) n k} h\left(x_{p+1-k}\right)
$$

for $x=\left(x_{1}, \cdots, x_{p}\right) \in W_{n}^{(1, k)}$ with $k \in\{1, \cdots, p-1\}$. Thus, $H_{1}(\cdot)$ : = $H(\cdot, 1)$ is equivariant on $W_{n}^{(1)}$.

(b) Let $d_{1}: W_{n}^{(1)} \times[0,1] \rightarrow(0,2 \pi)$,

$$
d_{1}(x, t):=\arg \left(\frac{H\left(\varphi_{3, p}(x), t\right)}{H(x, t)}\right) \text { for } \quad(x, t) \in W_{n}^{(1)} \times\{0,1\}
$$

and

$$
d_{1}(x, t):=t d_{1}(x, 1)+(1-t) d_{1}(x, 0) \text { for }(x, t) \in W_{n}^{(1)} \times(0,1) .
$$

Observe that we used here the fact that for $x=\left(x_{1}, \cdots, x_{p}\right) \in W_{n}^{(1)}$ we have $x_{2} \neq x_{1}$, which implies $H\left(\varphi_{3, p}(x), 0\right)=h\left(x_{2}\right) \neq h\left(x_{1}\right)=H(x, 0)$. Now we can define

$$
H(x, t):=H\left(\varphi_{3, p}^{p-k}(x), t\right) \prod_{m=1}^{k} e^{i d_{1}\left(\varphi_{3, p}^{p-m}(x), t\right)}
$$


for $(x, t) \in W_{n}^{(1, k)} \times(0,1), k \in\{1, \cdots, p-1\}$.

(c) $H$ is now given in particular on $\left(W_{n}^{(1)} \times[0,1]\right) \cup\left(W_{n}^{(2,0)} \times\{0\}\right)$. By a well known homotopy extension theorem (cf. [3], p. 14), we can extend $H$ continuously to the set $\left(W_{n}^{(1)} \cup W_{n}^{(2,0)}\right) \times[0,1]$ such that $H\left(\left(W_{n}^{(1)} \cup W_{n}^{(2,0)}\right) \times[0,1]\right) \subset S^{1}$. Furthermore, we can define for $x \in$ $W_{n}^{(2, k)}$ with $k \in\{1, \cdots, p-1\}$ :

$$
H(x, 1):=f^{k}\left(H\left(\varphi_{3, p}^{p-k}(x), 1\right)\right)=e^{(2 \pi i) / p) n k} H\left(\varphi_{3, p}^{p-k}(x), 1\right) .
$$

(d) Let $d_{2}:\left(W_{n}^{(1)} \cup W_{n}^{(2)}\right) \times[0,1] \rightarrow(0,2 \pi)$ be defined analogously to $d_{1}$. Since, for $x \in W_{n}^{(1)} \cup W_{n}^{(2)},\left(a_{1}, \cdots, a_{p}\right) \in T_{x}$ and $s \in\{1, \cdots, p\}$, we have

$$
\left|\frac{2 \pi}{3} \sum_{m=1}^{s} r\left(a_{m}, a_{m^{+}}\right)-\sum_{m=1}^{s} d_{2}\left(\varphi_{3, p}^{m-1}(x), 0\right)\right| \leqq \frac{2 \pi}{3},
$$

which implies

$$
\sum_{m=1}^{p} d_{2}\left(\varphi_{3, p}^{m-1}(x), 0\right)=\frac{2 \pi}{3} \sum_{m=1}^{p} r\left(a_{m}, a_{m}+\right)=2 \pi n,
$$

it follows for every $(x, t) \in\left(W_{n}^{(1)} \cup W_{n}^{(2)}\right) \times[0,1]$ that

$$
\begin{aligned}
\sum_{m=1}^{p} d_{2} & \left(\varphi_{3, p}^{p-m}(x), t\right) \\
& =t \sum_{m=1}^{p} d_{2}\left(\varphi_{3, p}^{p-m}(x), 1\right)+(1-t) \sum_{m=1}^{p} d_{2}\left(\varphi_{3, p}^{p-m}(x), 0\right) \\
& =t \sum_{m=1}^{p} \frac{2 \pi}{p} n+(1-t) 2 \pi n=2 \pi n
\end{aligned}
$$

Hence, for $(x, t) \in W_{n}^{(1)} \times[0,1]$ and $k \in\{1, \cdots, p-1\}$, we have

$$
\begin{aligned}
& H\left(\varphi_{3, p}^{p-k}(x), t\right) \prod_{m=1}^{k} e^{i d_{2}\left(\varphi_{3, p}^{p-m}(x), t\right)} \\
& \quad=H(x, t) \prod_{m=k+1}^{p} e^{i d_{1}\left(\varphi_{3, p}^{p-m}(x), t\right)} \prod_{m=1}^{k} e^{i d\left(\varphi_{2}^{p-3}(x), t\right)} \\
& =H(x, t) \prod_{m=k+1}^{p} e^{i d_{2}\left(\varphi_{3, p}^{p-m}(x), t\right)} \prod_{m=1}^{k} e^{i d_{2}\left(\varphi_{3, p}^{p-m}(x), t\right)} \\
& =H(x, t) \prod_{m=1}^{p} e^{i d_{2}\left(\varphi_{3, p}^{p-m}(x), t\right)}=H(x, t) e^{i 2 \pi n}=H(x, t) .
\end{aligned}
$$

This justifies the definition

$$
H(x, t):=H\left(\varphi_{3, p}^{p-k}(x), t\right) \prod_{m=1}^{k} e^{i d_{2}\left(\varphi_{3, p}^{p-m}(x), t\right)}
$$

for $(x, t) \in W_{n}^{(2, k)} \times(0,1), k \in\{1, \cdots, p-1\}$.

(e) To obtain $H$ on $\left(W_{n}^{(1)} \cup W_{n}^{(2)} \cup W_{n}^{(3,0)}\right) \times[0,1]$, we apply the same homotopy extension theorem as in (c). Finally, we obtain 
$P: W_{n} \rightarrow S^{1}$ by

$$
P(x):=\left\{\begin{array}{l}
H(x, 1) \text { for } x \in W_{n}^{(1)} \cup W_{n}^{(2)} \cup W_{n}^{(3,0)} \\
f^{k}\left(H\left(\varphi_{3, p}^{p-k}(x), 1\right)\right) \text { for } x \in W_{n}^{(3, k)} \text { with } k \in\{1, \cdots, p-1\} .
\end{array}\right.
$$

For $k \geqq 4$ and $p \geqq 3$, only estimates of $g\left(L_{k, p}, \varphi_{k, p}\right)$ are known, which seem to be not best possible in most cases. However, we can prove a new result, which yields, in conjunction with Theorem 2, a slight improvement of Satz 10 in [8]:

TheOREM 5. Let $p \geqq 3$ be a prime number and $k \in\{3,4,5, \cdots\}$. Then we have

$$
g\left(L_{k, p}, \varphi_{k, p}\right)=g\left(\widetilde{L}_{k, p}, \varphi_{k, p}\right) \leqq \frac{p-1}{2}(k-3)+\left\{\begin{array}{lll}
1 & \text { if } & p=3 \\
2 & \text { if } & p \geqq 5
\end{array} .\right.
$$

Proof. Let $M_{i}:=\left\{\left(x_{1}, \cdots, x_{p}\right) \in \widetilde{L}_{k, p} \mid x_{1} \in \Delta_{k-1: i}\right\}$ and $F_{i}:=$ $\bigcup_{j=0}^{p-1} \varphi_{k, p}^{j}\left(M_{i}\right)(i=1, \cdots, k-3)$, and let

$$
G:=\widetilde{L}_{k, p} \cap\left(\bigcup_{j=k-2}^{k} \Delta_{k-1 ; j}\right)^{p} .
$$

Then we have

$$
\widetilde{L}_{k, p}=\bigcup_{i=1}^{k-3} F_{i} \cup G
$$

As a consequence of Theorems 2 and 4 , we have

$$
g\left(G, \varphi_{k, p}\right) \leqq\left\{\begin{array}{lll}
1 & \text { if } & p=3 \\
2 & \text { if } & p \geqq 5
\end{array} .\right.
$$

Furthermore, in the proof of Satz 10 in [8], it was shown that $g\left(F_{i}, \varphi_{k, p}\right) \leqq(p-1) / 2$. It follows that

$$
\begin{aligned}
g\left(\widetilde{L}_{k, p}, \varphi_{k, p}\right) & \leqq \sum_{i=1}^{k-3} g\left(F_{i}, \varphi_{k, p}\right)+g\left(G, \varphi_{k, p}\right) \\
& \leqq \frac{p-1}{2}(k-3)+\left\{\begin{array}{lll}
1 & \text { if } & p=3 \\
2 & \text { if } & p \geqq 5
\end{array}\right.
\end{aligned}
$$

\section{REFERENCES}

1. K. Borsuk, Drei Sätze über die n-dimensionale euklidische Sphäre, Fund. Math., 20 (1933), 177-190.

2. J. Dugundji, A duality property of nerves, Fund. Math., 59 (1966), 213-219.

3. Sze-Tsen Hu, Homotopy Theory, Academic Press, New York and London, 1959.

4. M. A. Krasnosel'skii, On special coverings of a finite-dimensional sphere, Doklady Akad. Nauk SSSR, 103 (1955), 961-964. (Russian)

5. L. Ljusternik and L. Schnirelmann, Topological methods in variational problems, 
Issledowatel'skii Institut Mathematiki i Mechaniki pri J. M. G. U., Moscow, 1930. (Russian)

6. A. S. Švarc, Some estimates of the genus of a topological space in the sense of Krasnosel'skǐ, Uspehi Mat. Nauk, 12 (1957), no. 4 (76), 209-214. (Russian)

7. — The genus of a fiber space, Trudy Moskov. Mat. Obšč., 10 (1961), 217-272 and 11 (1962), 99-126; (Russian), English translation in Amer. Math. Soc., Translat., II. Ser., 55 (1966), 49-140.

8. H. Steinlein, Borsuk-Ulam Sätze und Abbildungen mit kompakten Iterierten, Habilitationsschrift, University of Munich, 1976.

9. Chung-Tao Yang, On theorems of Borsuk-Ulam, Kakutani-Yamabe-Yujobô and Dyson, I, Ann. Math., 60 (1954), 262-282.

Received October 30, 1978. This research was started, when I was visiting the Université de Montréal in fall 1977. I would like to thank Prof. Granas and the mathematical institute for their kind hospitality.

MATHEMATISCheS InstituT

DER UNIVERSITÄT MÜNCHEN

THERESIENSTR. 39

D-8000 MÜnchen 2, Germany 


\section{PACIFIC JOURNAL OF MATHEMATICS}

EDITORS

DONALD BABBITT (Managing Editor)

University of California

Los Angeles, California 90024

Hugo RossI

University of Utah

Salt Lake City, UT 84112

C. C. MOORE and ANDREW OGG

University of California

Berkeley, CA 94720

\section{J. DUGUNDJI}

Department of Mathematics University of Southern California Los Angeles, California 90007

R. Finn and J. Milgram Stanford University

Stanford, California 94305

\section{ASSOCIATE EDITORS}
E. F. BECKENBACH
B. H. Neumann
F. WOLF
K. YoSHIDA

\section{SUPPORTING INSTITUTIONS}

UNIVERSITY OF BRITISH COLUMBIA

CALIFORNIA INSTITUTE OF TECHNOLOGY

UNIVERSITY OF CALIFORNIA

MONTANA STATE UNIVERSITY

UNIVERSITY OF NEVADA, RENO

NEW MEXICO STATE UNIVERSITY

OREGON STATE UNIVERSITY

UNIVERSITY OF OREGON
UNIVERSITY OF SOUTHERN CALIFORNIA

STANFORD UNIVERSITY

UNIVERSITY OF HAWAII

UNIVERSITY OF TOKYO

UNIVERSITY OF UTAH

WASHINGTON STATE UNIVERSITY

UNIVERSITY OF WASHINGTON 


\section{Pacific Journal of Mathematics}

\section{Vol. 83, No. 1 \\ March, 1979}

Richard Neal Ball, Topological lattice-ordered groups ............... 1

Stephen Berman, On the low-dimensional cohomology of some

infinite-dimensional simple Lie algebras .................. 27

R. P. Boas and Gerald Thomas Cargo, Level sets of derivatives ......... 37

James K. Deveney and John Nelson Mordeson, Splitting and modularly

perfect fields......................................

Robert Hugh Gilman and Ronald Mark Solomon, Finite groups with small

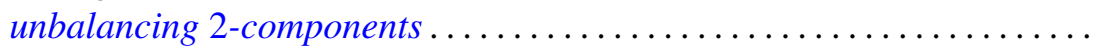

George Grätzer, Andras Hajnal and David C. Kelly, Chain conditions in free products of lattices with infinitary operations..................

Benjamin Rigler Halpern, Periodic points on tori ..................

Dean G. Hoffman and David Anthony Klarner, Sets of integers closed under

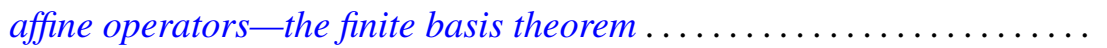

Rudolf-Eberhard Hoffmann, On the sobrification remainder ${ }^{s} X-X \ldots \ldots$

Gerald William Johnson and David Lee Skoug, Scale-invariant

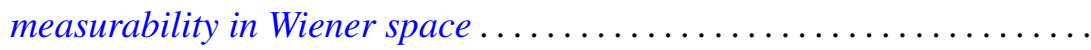

Michael Keisler, Integral representation for elements of the dual of

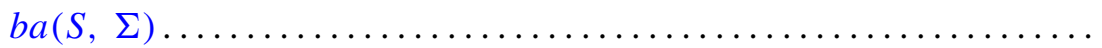

Wayne C. Bell and Michael Keisler, A characterization of the representable Lebesgue decomposition projections ................

Wadi Mahfoud, Comparison theorems for delay differential equations ...

R. Daniel Mauldin, The set of continuous nowhere differentiable functions .

Robert Wilmer Miller and Mark Lawrence Teply, The descending chain condition relative to a torsion theory...

Yoshiomi Nakagami and Colin Eric Sutherland, Takesaki's duality for regular extensions of von Neumann algebras ........ .

William Otis Nowell, Tubular neighborhoods of Hilbert cube manifolds ...

Mohan S. Putcha, Generalization of Lentin's theory of principal solutions of word equations in free semigroups to free product of copies of positive reals under addition

Amitai Regev, A primeness property for central polynomials . ...

Saburou Saitoh, The Rudin kernels on an arbitrary domain. . .

Heinrich Steinlein, Some abstract generalizations of the

Ljusternik-Schnirelmann-Borsuk covering theorem . . . 